\title{
Prevención de infección nosocomial mediante medidas implantadas en un hospital de Andalucía por bacterias resistentes a carbapenémicos
}

\author{
Francisco Javier Antúnez Rodríguez, Ana García Duque y \\ Adrián Jiménez Salido \\ Hospital Universitario Virgen Macarena, Sevilla (España)
}

\begin{abstract}
La aparición y expansión de bacterias productoras de carbapenemasas constituye una amenaza global de infecciones sin tratamiento eficaz. Estas bacterias suele presentar resistencia a todos los antimicrobianos de primera línea, produciendo infecciones muy difíciles de tratar. Los carbapenemas son de amplio espectro y potencia, y debido a eso se utiliza en pacientes con infecciones graves, incluso cuando el riesgo a priori de tener una infección por bacterias multirresistentes (MDR) es escaso. Existe una gran tendencia a mantener los mismos antibióticos que se inició hasta finalizar el tratamiento, aunque el paciente carezca de factores de riesgo para tener una infección por bacterias MDR. Todo esto produce un aumento evitable del consumo de antibióticos con carbapenemas. Nuestro objetivo es analizar si la incidencia de infección/colonización por pseudomona aeruginosa en un hospital de Andalucía se reduce tras la implantación de un conjunto de medidas persuasivas dirigidas a la optimización de los tratamientos con carbapenemas y analizar el consumo de carbapenemas. El método utilizado en un estudio cuasi experimental ecológico de series temporales de un hospital de Andalucía y evaluación trimestral de indicadores.
\end{abstract}

Palabras clave: Infección nosocomial, bacterias Gram negativas, multirresistentes, antibiótico, carbapenémico, hospital.

Measures to Nosocomial Infections Prevention by Carbapenems-resistant Bacteria in an Andalusian Hospital. The appearance and expansion of carbapenem producing bacteria constitute a global threat of infections without effective treatment. These bacteria usually offer resistance to all antimicrobial of the first-line, producing infections that are very difficult to treat. carbapenem are antimicrobial of wide spectre and high potenty, and due to that they're used in patients with serious infections, even when a prior risk to have infection by multi-resistant bacteria (MDR) is low. A great tendency exists to keep the same antibiotic from the beginning of treatment until its end, despite the lack of risk factors to have an infection by bacteria MDR. All this produces an avoidable increase of carbapenem antibiotic consumption. Our objective is to analyze if the infection incidence/colonization by Pseudomona aeruginosa in an Andalusian hospital is reduced after the implantation of a set of persuasive measures directed to the optimization of the treatments with carbapenem and to analyze the consumption of carbapenem antibiotics. The method used in an quasiexperimental-ecological study of temporal series from an Andalusian hospital and a quarterly evaluation of indicators.

Keywords: Nosocomial infection, Gram negative bacteria, multi-resistant, antibiotic, carbapenem, hospital.

Correspondencia: Francisco Javier Antúnez Rodríguez. Facultad de Medicina de la Universidad de Sevilla. Departamento de Microbiología, sótano. Avda. Sánchez Pizjuan, s/n. C.P.: 41009. Sevilla (España).E-mail: fjatnunez@hotmail.com 
La aparición de los antibióticos en su uso clínico fue un hito importantísimo para poder avanzar en la medicina, ya sea de forma directa curando distintos tipos de infecciones, como de forma indirecta favoreciendo un avance dentro de los distintos procesos terapéuticos asociados a infecciones bacterianas. La utilización de los antimicrobianos se ve muy favorecida en los casos de infecciones graves, como pueden ser sepsis grave y/o shock séptico, donde una utilización adecuada de dichos antibióticos se relaciona con una importante bajada de los casos de mortalidad. De hecho es la intervención terapéutica con mayor impacto conocida en la actualidad.

A pesar de todo esto, durante el paso del tiempo utilizando antibióticos, se ha podido observar como las bacterias se han ido mejorando ante la sensibilidad antimicrobiana volviéndose así resistentes a determinados antibióticos a través de distintos mecanismos adquiridos de resistencia. Esta resistencia de los microorganismos a los antimicrobianos se ha ido resolviendo con la nueva creación de distintos antibióticos, pero esa solución se ve cada vez a disminuida debido a la poca perspectiva de la creación de nuevos antibióticos, llegando casi al descubrimiento de casi todas las posibilidades, afirmando así que el modelo establecido hasta el momento no puede mantenerse. Cada vez aparecen más muertes ocasionadas por microorganismos resistentes a antibióticos denominándose así por algunos autores como "crisis antibiótica", anticipándose también a una "era post-antibiótica", donde es evidente la poca disponibilidad de antibióticos que garantice un tratamiento eficaz y adecuado.

Todo el problema ocasionado por la resistencia bacteriana no es nuevo de ahora, sino que se ha estado avisando por toda la comunidad científica durante bastante tiempo, con lo que ha llevado a la implantación de unas estrategias enfocadas a disminuir dicho problema. Estas estrategias van enfocadas a favorecer y dar rapidez en el desarrollo y comercialización de nuevos antibióticos eficientes para los microorganismos más patógenos, optimizar el control sanitario en los centros de salud y hacer que la vida útil de los antibióticos sea más larga obteniéndose así mejores resultados en infecciones clínicas graves.

Un uso adecuado de antibióticos es importante no solo para la prolongación de su vida útil, sino además de garantizar la eficacia en casos de infecciones graves. También es importante resaltar que la optimización del uso de antibióticos debe de disminuir la aparición de nuevos casos de resistencia. Debido a que los antibióticos son uno de los medicamentos más utilizados en el hospital, incluso sabiendo que esta utilización es segura, se ha demostrado en un estudio español que el $20 \%$ de los casos de urgencias están relacionados directamente con el uso inadecuado de antibióticos, siendo casi el doble de los medicamentos considerados antes como "alto riesgo" (insulina, anticoagulantes orales). En las últimas décadas, hemos asistido a un aumento progresivo en la diseminación de bacterias gramnegativas resistentes a múltiples antimicrobianos (World Health Organization, 2014). Hasta hace pocos años, la mayoría de las bacterias 
gramnegativas eran sensibles a antimicrobianos del tipo carbapenemas, constituido como tratamiento de elección en la mayoría de las infecciones. Sin embargo, la aparición y expansión de bacterias productoras de carbapenemasas, en especial enterobacterias y Acinetobacter baumannii, o resistente a carbapenemas mediante otros mecanismos, especialmente pseudomona aeruginosa, constituye una amenaza global de infecciones sin tratamiento eficaz. La propagación de enterobacterias productoras de carbapenemasas (EPC), de reciente aparición, es bastante preocupante. Estas bacterias suelen presentar resistencia a todos los antimicrobianos de primera línea, produciendo infecciones muy difíciles de tratar. Los pacientes que son infectado con estas enterobacterias suelen ser portadores, transformándose en reservorio de futuros brotes (Tängdén, 2015).

La presión antibiótica tiene una gran importancia, directa o indirectamente, en la expansión de los bacilos gramnegativos resistentes a carbapenemas (BGNRC). El uso de carbapenemas en un medio, es directamente proporcional a la aparición de bacterias resistentes, como demuestran los factores de riesgo detectados para diversos brotes de A. baumannii (Liu et al., 2014) P. aeruginosa y EPC (Nakamura et al., 2013).

Los carbapenemas son de amplio espectro y potencia y debido a eso se utiliza en pacientes con infecciones graves, incluso cuando el riesgo a priori de tener una infección por bacterias multirresistentes (MDR) es escaso. En el caso de tomar muestras para cultivo y cuyo resultado es positivo, en unas $48 \mathrm{~h}$ se puede ajustar el tratamiento para los aislados obtenidos, siendo esta medida una recomendación en los programas de optimización de antibióticos. Pero a pesar de dicha recomendación, existen una gran ausencia de cultivos que orienten para dicha simplificación y debido a esta ausencia hay una gran tendencia a mantener el mismo antibiótico que se inició hasta finalizar el tratamiento, aunque el paciente carezca de factores de riesgo para tener una infección por bacterias MDR (Gauzit et al., 2015). Todo esto produce un aumento evitable del consumo de antibióticos con carbapenemas, que varía según los hábitos de prescripción de cada centro.

Los programas de optimización del tratamiento antibiótico (PROA) han demostrado ser útiles en la racionalización el consumo de antimicrobianos (Cisneros, 2014). El programa PIRASOA, implantado desde enero de 2014 en todos los hospitales públicos andaluces y distritos de Atención Primaria de Andalucía, tiene la finalidad de reunir las intervenciones sobre la optimización en el uso racional de antimicrobianos con la información epidemiológica sobre bacterias multirresistentes.

Hasta la fecha, en Andalucía se han producido brotes aislados de infección por EPC en el 50\% de los hospitales y transmisión intercentros, como demuestran los datos del informe del Laboratorio de Referencia del PIRASOA (PIRASOA, 2016 ), que colocan a Andalucía en el nivel 3 de la clasificación de los eCDC, definido por "la existencia de más de un brote epidemiológicamente relacionado, confinado a hospitales 
que son parte de la red de referencia regional que sugieren la transmisión regional interinstitucional autóctona" (Control, 2011). Igualmente existe una incidencia variable de P. aeruginosa y A. baumannii MDR en los hospitales andaluces: 0.0-0.8/1000 estancias en el caso de P. aeruginosa y 0.0-0.3/1000 estancias en el caso de A. baumannii (PIRASOA, 2016).

En la actualidad, las actividades de PROA se basan en un programa educativo basado en asesorías no impositivas al azar, siguiendo el modelo descrito por Cisneros (2014), que permite a la vez conocer la proporción aproximada de tratamientos inapropiados y realizar intervención educativa.

\section{Objetivos}

- Analizar si la incidencia de infección/colonización por pseudomona aeruginosa en un hospital de Andalucía, cuyo consumo de carbapenemasas es alto, se reduce tras la implantación de un conjunto de medidas persuasivas dirigidas a la optimización de los tratamientos con carbapenemas.

- Analizar el consumo de carbapenemas.

- Evaluar la incidencia de infección/colonización por P. aeruginosa MDR.

- Definir la diseminación clonal y los mecanismos de resistencia de las P. aeruginosa MDR.

\section{MÉTODO}

\section{Diseño}

Estudio cuasi experimental ecológico de series temporales de un hospital de Andalucía. Ámbito: El periodo de estudio es retrospectivo de 4 trimestres (septiembre 2016-septiembre 2017).

\section{Procedimiento}

La intervención será la implantación de un "bundle" de medidas persuasivas dirigido a optimizar los tratamientos con carbapenemas. Lo coordinará el equipo responsable de actividades de PROA en el marco del PIRASOA del hospital. El "bundle" constará de cuatro elementos:

a) Documentación propia estableciendo las indicaciones institucionales de uso de carbapenemas: el hospital elegirá una guía de referencia para el tratamiento de los principales síndromes infecciosos, ajustadas a la ecología local.

b) Asesorías clínicas no impositivas: las realizará el equipo de profesionales que hace habitualmente asesorías como parte de las actividades del PROA. Los médicos prescriptores serán seleccionados semanalmente a partir de los datos proporcionados por 
la Farmacia hospitalaria del centro. El método para la realización de las asesorías será el descrito por Cisneros (Cisnero, 2014).

c) Aviso automático con indicaciones de simplificación a las 48 horas del inicio del tratamiento con carbapenemas, recordando las indicaciones de simplificación.

d) Análisis de resultados: evaluación trimestral de indicadores.

e) Retroalimentación de la información: se realizará un análisis de indicadores de consumo trimestral del hospital y se transmitirá a todos sus miembros.

\section{Análisis de resultados}

Evaluación trimestral de indicadores. Indicadores del análisis (PIRASOA, Programa integral de prevención y control de las infecciones relacionadas con la asistencia sanitaria y uso apropiado de los antimicrobianos. 2014):

- Objetivo 1: Realizar el estudio del mecanismo de resistencia a carbapenemas mediante técnicas fenotípicas (fenotipo de resistencia, actividad de diferentes inhibidores, Carba NP test, etc) y genotípicas (PCR y secuenciación de los genes involucrados) de todos los aislados de P. aeruginosa resistente a carbapenemas que se produzca en un hospital Andalucía.

- Objetivo 2: Higiene de manos. Formación y monitorización del cumplimiento de las normas donde se ha producido y repetidos casos de resistencia bacteriana a carbapenémicos.

- Objetivo 3: Optimización del uso de los antimicrobianos. Coordinación con el equipo de PROA para la evaluación diagnóstica y terapéutica de los casos y la aplicación de medidas específicas de optimización del tratamiento antimicrobiano a la situación epidemiológica.

- Objetivo 4: Cumplimiento de los indicadores PROA (PIRASOA, 2014).

- Objetivo 5: Consumo de imipenem, meropenem y ertapenem expresados en DDD/1000estancias/día.

\section{RESULTADOS}

En un hospital de Andalucía se recogieron un total de 78 aislados P. aeruginosa resistentes a antibióticos carbapenémicos durante el periodo del cuarto trimestre del 2016, primer, segundo y tercer trimestre del 2017, siendo 25, 21, 16 y 16 aislados respectivamente. De estos 78 aislados resistente a carbapenémicos, sólo 9 eran productores de carbapenemasas, 7 perteneciente al cuarto trimestre de 2016, 2 al primer, 0 al segundo y 0 al tercer trimestre de 2017. 
Gráfica 1. Aislados totales encontrados en un hospital de Andalucía.

Resistentes Carbapenémicos

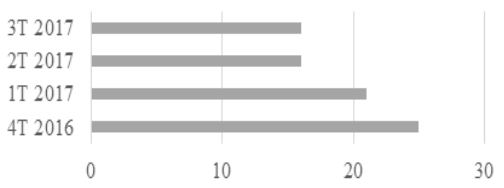

A todos los aislados pseudomona eruginosa resistentes a carbapenémicos se le hicieron un estudio genotípico mediante PCR con la finalidad de detectar los genes KPC, IMP, VIM, NDM u OXA-48, determinantes codificantes de carbapeneasmasas que originan resistencia a carbapenémicos, obteniéndose sólo 7 aislados productores de enzimas del grupo VIM y 2 aislados productores de IMP.

Gráfica 2. Aislados resistentes productores de carbapenémicas y otros mecanismos de resistencia

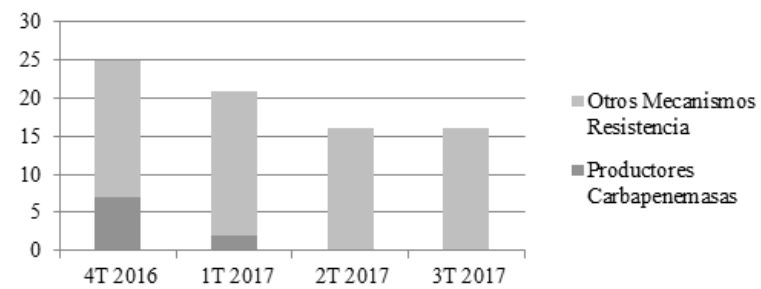

De este total aparecieron 5 aislados productores de VIM, 2 aislados productores de IMP y todos fueron negativos para KPC, NDM, OXA-48 durante el cuarto trimestre de 2016; 2 productores de VIM y el resto negativos para los otros genes durante los aislados recibidos en el primer trimestre de 2017 y ninguno más durante los dos trimestres restantes.

Gráfica 3. Distintos tipos de productores de carbapenemasas encontrados en los aislados

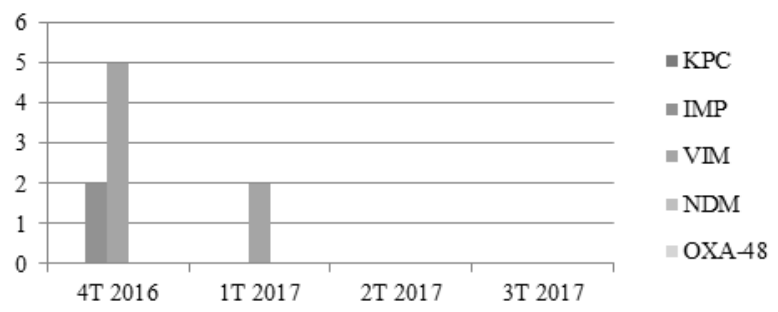


Gráfica 4. Totales Productores de carbapenemasas

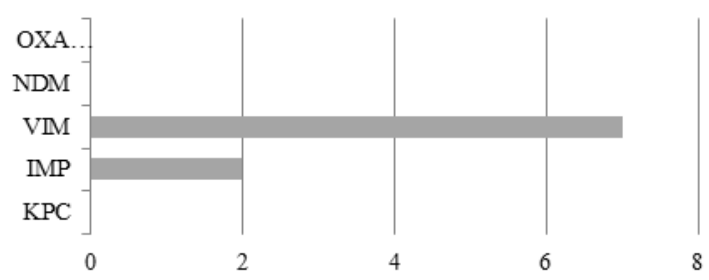

Además también se recogen las dosis diarias definidas (DDD) por cada 1000 estancias por día de antibióticos carbapenémicos (ertapenem, imipenem y meropenem) del mismo hospital, cuyos datos están publicados en el programa PIRASOA (PIRASOA, Informe cuarto trimestre e Iras anual. 2016; Servicio Andaluz de Salud, 2017).

También se han tenido en cuenta para este estudio el cumplimiento de los indicadores PROA, medidas higiénicas de manos y tasa de uso inadecuado de antimicrobianos recogido por el programa PIRASOA durante dicho periodo (PIRASOA, Informe cuarto trimestre e Iras anual. 2016; Servicio Andaluz de Salud, 2017) siendo para el cumplimiento de indicadores PROA el $100 \%$ en todos los trimestres estudiados, para el cumplimiento de medidas higiénica de manos del 83.13\%, 70.53\%, 57.09\%, $55.71 \%$ para el $4^{\circ}$ trimestre de 2016 y primero segundo y tercer trimestre de 2017 respectivamente; y por último también se ha tenido en cuenta la tasa de uso inadecuado de antimicrobianos, siendo $48.75 \%$ para el cuarto trimestre de 2016 y $28.71 \%, 23.98 \%$, $41.18 \%$ para el primer, segundo, tercer trimestre de 2017.

Gráfica 5. Cumplimiento de distintos parámetros PROA

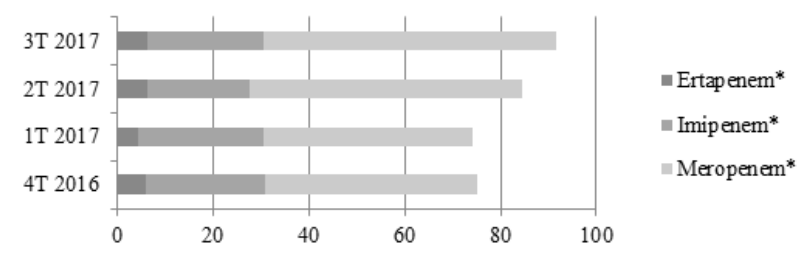

Gráfica 6. Cumplimiento de distintos parámetros PROA

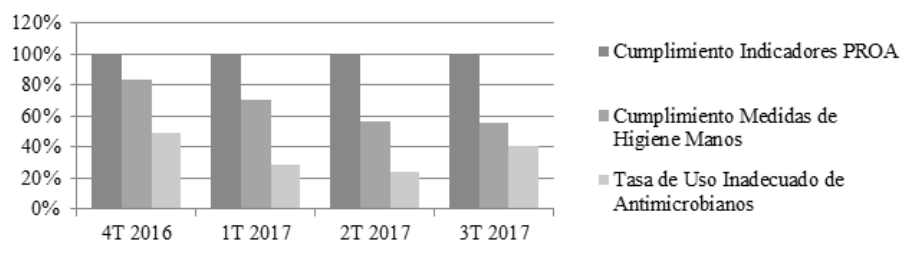


Gráfica 7. Consumo total de carbapenémicos en el periodo estudiado

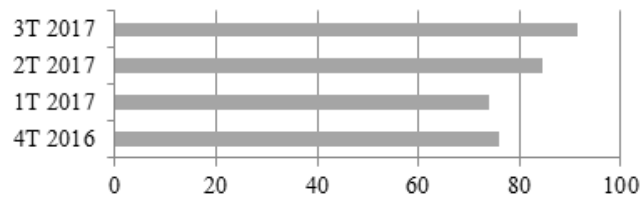

Tabla 1. Consumo total de carbapenémicos en el periodo estudiado DDD (Dosis Diaria Definida)/1000 estancias/día

\begin{tabular}{ccccc}
\hline & 4T 2016* & 1T 2017* & 2T 2017* & 3T 2017* \\
\hline Ertapenem & 5.73 & 4.38 & 6.25 & 6.26 \\
\hline Imipenem & 26.16 & 26.14 & 21.2 & 24.2 \\
\hline Meropenem & 44.13 & 43.61 & 56.97 & 61.21 \\
\hline
\end{tabular}

\section{DISCUSIÓN Y CONCLUSIONES}

De los 78 aislados P. eruginosa recogidos resistentes a carbapenemas, sólo 9 (12\%) aislados eran productores de carbapenemasas y el resto, 69 aislados, eran resistentes a carbapenémicos, pero no productores de las carbapenemasas estudiadas (KPC, IMP, VIM, NDM y OXA-48). Esto nos indica que tendrían otros mecanismos de resistencia a dichos antimicrobianos, probablemente relacionados con pérdida $\mathrm{O}$ disfunción de porinas junto con la activación de bombas de flujo.

De los 78 aislados resistente a carbapenemas, 25 de ellos corresponden al cuarto trimestre de 2016, 21 al primer trimestre de 2017, 16 al segundo y otros 16 al tercer trimestre de 2017. Aquí se observa una disminución notable de la presencia de aislados resistentes a carbapenémicos.

De los 9 aislados encontrados como productores de carbapenemasa, siete (78\%) de ellos eran positivos para VIM y dos (22\%) de ellos eran productores de IMP. Encontrando cinco VIM y dos IMP en el cuarto trimestre de 2016 y dos VIM en el primer trimestre de 2017; dejando el resto de los trimestres sin la presencia de ningún otro aislado productor de carbapenemasas. De esta forma se ve una disminución total en dicho periodo de aislados productores de carbapenemasas.

En cuanto a las dosis diarias definidas (DDD) por cada 1000 estancias por día, el consumo de antimicrobianos carbapenémicos totales ha ido subiendo en los cuatro trimestres estudiados, aumentando el DDD/1000 estancias/día de ertapenem y meropenem en los cuatro trimestres y disminuyendo un poco el de imipenem.

Respecto al cumplimiento de los indicadores PROA (PIRASOA, 2014), se ha mantenido al $100 \%$ durante todos los trimestres. La tasa de uso inadecuado de antimicrobianos ha ido disminuyendo en dicho periodo siendo $48.75 \%, 28.71 \%, 23.98 \%$ durante el cuarto trimestre de 2016, primer y segundo trimestre de 2017 respectivamente y teniendo un repunte en el tercer trimestre de 2017 con un $41.18 \%$. Esto nos indica que 
hubo un aumento de consumo de antimicrobianos de forma inadecuada durante el tercer trimestre de 2017, coincidiendo con un aumento del consumo total de carbapenémicos. Estos datos podrían suponer un aumento de aislados resistentes a carbapenémicos durante el cuarto trimestre, que habría que comprobar con dichos datos.

Respecto al cumplimiento de las medidas higiénicas de manos, donde se define como formación y, monitorización del cumplimiento en el área donde se ha producido el caso y retroalimentación directa y periódica de los resultados, podemos decir que ha ido disminuyendo durante los cuatro trimestres. Esta disminución coincide con la disminución de casos de aislados resistentes a antimicrobianos carbapenémicos. Esto apunta que podemos decir que la aparición de aislados resistente a carbapenémicos no está directamente relacionada con este índice, es decir, puede influir en su propagación de las bacterias, pero no en la aparición de nuevos casos de resistentes, con los datos estudiados y obtenidos.

\section{REFERENCIAS}

Cisneros, J.M., Neth, O., Gil-Navarro, M.V., Lepe, J.A., Jiménez-Parrilla, F., Cordero, E., Rodríguez-Hernández, M.J., Amaya-Villar, R., Cano, J., Gutiérrez-Pizarraya, A., GarcíaCabrera, E., y Molina, J. (2014). Global impact of an educational antimicrobial stewardship programme on prescribing practice in a tertiary hospital centre. Clinical Microbiology and Infection, 20(1), 82-88.

Control, E.C. (2011). Risk assessment on the spread of carbapenemase-producing Enterobacteriaceae (CPE) through patients transfer between healthcare facilities, with especial emphasis on crossborder transfer. Stockholm: ECDC.

Evans, B.A., Hamouda, A., y Amyes, S.G. (2013). The rise of carbapenem-resistance: Acinetobacter baumannii. Phrmaceutical Design, 19, 223-238.

Gauzit, R., Pean, Y., Alfandari, S., Bru, J.P., Bedos, J.P., (2015.). Carbapenem use in French hospitals: A nationwide survey at the patient level. International Journal Antimicrobiology Agents, 46(6), 707-712.

Liu, Q., Li, W., Feng, Y., y Tao, C. (2014). Efficacy and Safety of Polymyxins for the Treatment of Acinectobacter baumannii Infection: A Systematic Review and Meta-Analysis. PLos One, 9(6), e98091.

Marya, D.Z.M. (2016). Secular trends in Acinetobacter baumannii resistance in resiratory and blood stream specimens in the United States, 2003 to 2012: A survey study. Journal Hospital Medical, 11(1), 21-26.

Nakamura, A. et al. (2013). Meropenem as predictive risk factor for isolation of multidrugresistant Pseudomonas aeruginosa. Journal Hospiotal Infection, 83(2), 153-155.

PIRASOA (2016). Informe cuarto trimestre e Iras anual. Sevilla: Servicio Andaluz de Salud.

PIRASOA (s.f.). Programa integral de prevención y control de las infecciones relacionadas con la asistencia sanitaria y uso apropiado de los antimicrobianos.

PIRASOA, A.M. (s.f.). Indicadores de proceso y estructura programas PROA.

PIRASOA, I. (s.f.). Programa integral de prevención, control de las infecciones relacionadas con la asistencia sanitaria, y uso apropiado de los antimicrobianos. 
Qin, W. et al. (2014). Hospital clonal dissemination of Enterobacter aerogenes producing carbapenemase KPC-2 in a Chinese teaching hospital. Journal Medical Microbiology. 63(2), 222-228.

Servicio Andaluz de Salud, C. d. (2017). Informe segundo trimestre PIRASOA.

Servicio Andaluz de Salud, C. d. (2017). Informe tercer trimestre PIRASOA.

Servicio Andaluz de Salud, C. d. (s.f.). Informe primer trimestre PIRASOA.

Servico Andaluz de Salud, C. d. (2017). Informe primer, segundo y tercer trimestre del Programa PIRASOA.

Tängdén, T. et al. (2015). Global dissemination of extensively drug-resisitant carbapenemaseproducing Enterobacteriaceae: clinical perspectives on detección, tretment and infection control. Journal International Medical, 277(5), 501-512.

World Health Organization (2014). Antimicrobial resistance: global reporto on surveillance. Geneva: WHO.

Recibido: 08 de julio de 2018

Recepción Modificaciones: 21 de julio de 2018

Aceptado: 26 de julio de 2018 